\title{
On the Eternal Role of Planck Scale in Machian Cosmology
}

\author{
U. V.S. Seshavatharam ${ }^{1}$ and S. Lakshminarayana ${ }^{2}$ \\ ${ }^{1}$ Honorary Faculty, I-SERVE, S. No-42, Hitex Road, Hitech City, Hyderabad-84, Telangana, India. \\ ${ }^{2}$ Department of Nuclear Physics, Andhra University, Visakhapatnam-03, AP, India. \\ Email(s): seshavatharam.uvs@gmail.com (and) sln@auvsp.edu.in
}

\begin{abstract}
Considering Planck mass as a baby universe and evolving universe as a growing Planck ball, a hypothetical spherical model of cosmology can be developed. In addition, by considering the famous relation $G M=c^{2} R$, a Machian model of quantum cosmology can also be developed. Proceeding further, in all directions, cosmic expansion velocity can be referred to 'from and about' the baby universe. This hypothetical scenario can be made versatile by adding a heuristic idea: apart from normal expansion, 'cosmic temperature seems to be redshifted by a factor $\left(\left(Z_{T}\right)_{t}+1\right) \cong \sqrt{1+\ln \left(H_{p l} / H_{t}\right)}$ where $\left(H_{p l}, H_{t}\right)$ represent Planck scale and time dependent Hubble parameters respectively.
\end{abstract}

Keywords: Planck scale; Mach's relation; quantum cosmology; critical density; ordinary matter; dark matter; thermal redshift; expansion velocity; Hubble's law;

\section{INTRODUCTION}

Replacing 'big bang' (Gamow 1948a,1948b, Abhas Mitra 2011, 2014) with a growing Planck ball and considering 'Mach's relation' (Patrick Grahn et al 2018; Annila 2012; Berman 2008a, 2009b; Arbab 2001a,2004b; Miyazaki 2001;) as a deep cosmic probe, in a hypothetical approach, an evolving model of quantum cosmology can be developed (Bojowald 2015, Arbab 2004, Padmanabhan 2006, Sivaram 2000). To make the model sensible, we assume that, apart from normal expansion, cosmic temperature is redshifted by a factor, $\sqrt{\gamma_{t}} \cong \sqrt{1+\ln \left(H_{p l} / H_{t}\right)} \cong\left(\left(Z_{T}\right)_{t}+1\right)$ where $\left(H_{p l}, H_{t}\right)$ represent Planck scale and time dependent Hubble parameters and $\left(Z_{T}\right)_{t}$ is the redshift associated with temperature $T_{t}$ about the baby universe. Considering the characteristic $\gamma_{t}$, current cosmic temperature and current Hubble parameter can be fitted and we make an attempt to estimate the current cosmic mass and radius. Proceeding further, in analogy with 'cosmic acceleration', in this paper, using $\gamma_{t}$, we proposed simple relations for a decreasing trend of total matter density with increasing trend of expansion velocity referred to 'from and about' the baby universe in all directions.

\subsection{Motivating Points}

1) Mach's principle (Einstein 1918) is one of the iconic principles underlying general theory of relativity and can be given a priority in developing a workable or unified model of cosmology.

2) Cosmic expansion, Lambda term, dark matter, cosmic temperature, inflation, cosmic acceleration and dark energy and vacuum energy are different concepts, by using which alternative models of GTR are emerging and are being extended in many ways. In this sequence, quantum cosmology can also be given some consideration.

3) Quantum cosmology is a wide range physical model intended for understanding the in-built cosmological quantum phenomena on small scale as well as large scale distances. So far, progress in this direction is very nominal and 'GTR' needs a serious review with reference to 'quantum cosmology'.

4) When universe is able to give birth to atoms, elementary parrticles and photons that show quantrum behaviour, universe can certainly be considered as a quantum gravitational object for ever.

5) What to quantize? How to quantize? When to quantize? and What to measure? etc. are some interesting questions in current quantum cosmology and need a special focus. In this context, cosmic temperature can be considered as a characteristic feature of quantum cosmology.

6) According to current notion, if the known laws of physics are extrapolated to the highest density regime, the result is a singularity which is typically associated with the Big Bang. In this context, Planck mass can be considered as a characteristic seed of the evolving universe and big bang can be replaced with an evolving Planck ball. Planck mass can be called as the 'baby Planck ball' or 'baby universe'.

7) With reference to particle physics, current technological limits on particle colliding energy, unidentified/unseen

Dedicated to Dr. Stephen William Hawking 
particles, unknown particle interactions and incomplete final unification scheme - to some extent, one can hopefully believe in the existence of dark matter (Rennan Barkana, 2018).

8) Basically, 'dark energy' was proposed for understanding cosmic acceleration. Careful analysis of improved supernovae data suggets that universe is coasting at constant veleocity and evidence for acceleration is only marginal (Neilsen et al. 2016, Lawrence H. Dam et al., 2017). In this context, now a days, a great debate has been initiated among mainstream copsmologists on the existence of dark energy (Balakrishna et al 2017, G'abor R'acz et al 2017, Lasha Berezhiani et al, 2017, Joel Smoller et al, 2017). According to a new study (Gong-Bo Zhao et al, 2017), the nature of dark energy is 'dynamic' and conceptually seems to deviate from the famous cosmological constant or vaccum energy. According to another new study (Deng Wang and Xin-He Meng, 2017), evidence for dynamical dark energy is very poor.

9) Density perturbations (Joel Smoller et al 2017) and interaction between dark mtter and baryons (Lasha Berezhiani et al 2017) seem to play a crucial role in understanding observed cosmic acceleration and need of introducing dark energy seems to be ad-hoc.

10) Even though redshift is an index of cosmic expansion, without knowing the actual galactic distances and actual galactic receding speeds, with $100 \%$ confidence level, it may not be possible to decide the absolute nature of cosmic expansion rate.

\section{ASSUMPTIONS, CONCEPTS AND BASIC RELATIONS}

\subsection{Nomenclatures}

1) $\quad\left(\Omega_{O M}\right)_{t}=$ Ratio of ordinary matter density to critical density.

2) $\quad\left(\Omega_{D M}\right)_{t}=$ Ratio of dark matter density to critical density.

3) $H_{t}=$ Hubble parameter and $H_{p l}=$ Planck scale Hubble parameter.

4) $\quad\left(V_{\exp }\right)_{t}=$ Cosmic expansion velocity from and about the baby universe or baby Planck ball.

5) $\quad\left(M_{O M}\right)_{t}=$ Cosmic ordinary mass content, $\left(M_{D M}\right)_{t}=$ Cosmic dark matter content.

6) $\quad\left(M_{O M}+M_{D M}\right)_{t} \cong M_{t}=$ Total matter content $=$ Total mass of evolving Planck ball.

7) $\quad R_{t}=$ Cosmic radius associated with $M_{t}=$ Radius of evolving Planck ball.

8) $T_{t}=$ Cosmic temperature and $\left(Z_{T}\right)_{t}=$ Redshift associated with $T_{t}$ about the baby universe.

9) $\quad\left(T_{c}\right)_{t}=\left(\frac{3 H_{t}^{2} c^{2}}{8 \pi G a}\right)^{\frac{1}{4}}=$ Critical temperature.

10) $\gamma_{t} \cong\left[1+\ln \left(\frac{H_{p l}}{H_{t}}\right)\right] \cong \sqrt{\frac{3 H_{t}^{2} c^{2}}{8 \pi G\left(a T_{t}^{4}\right)}}=$ Root of ratio of critical energy density and thermal energy density.

11) $\left(d_{g}\right)=$ Galactic distance from and about the baby universe or baby Planck ball.

12) $\left(v_{g}\right)=$ Galactic receding speed from and about the baby universe or baby Planck ball.

Note-1: For the above symbols, subscript 0 denotes current value and subscript $p l$ denotes Planck scale value.

\subsection{Proposed Assumptions}

With respect to our earlier publications (Tatum et al 2015, Seshavatharam \& Lakshminarayana 2017a, 2017b, 2018c, 2018d) in this paper we review the basic assumptions.

1) Planck scale and Mach's relation play a crucial role in entire cosmic evolution.

2) Apart from normal expansion, cosmic temperature is redshifted by a factor $\sqrt{\gamma_{t}} \cong \sqrt{1+\ln \left(H_{p l} / H_{t}\right)} \cong\left(\left(Z_{T}\right)_{t}+1\right)$.

3) Ordinary matter and dark matter, both, play a crucial role in estimating cosmic expansion velocity and there is no need to consider dark energy.

Note-2: If there is no redshift in cosmic temperature, at any stage of cosmic evolution, thermal energy density and critical energy density will always be equal in magnitude. It may also be noted that, proposed thermal redshift is with reference 
to the baby universe and currently believed notion of redshift is with reference to distance between earth and any receding galaxy.

Note-3: Based on these assumptions, from the beginning of Planck scale, a) With a 'decreasing' trend of total matter density ratio, cosmic expansion velocity can be shown to be increasing. b) With an 'increasing' trend of total matter density ratio, cosmic expansion velocity can be shown to be decreasing. c) With a constant trend of total matter density ratio, cosmic expansion velocity can be shown to be constant.

\subsection{Choosing the Magnitude of $H_{0}$}

1) As per the 2015 Planck data (Planck Collaboration 2015): $H_{0} \cong(67.31 \pm 0.96) \mathrm{km} / \mathrm{sec} / \mathrm{Mpc}$ and the present temperature of the $\mathrm{CMB}$ radiation is, $T_{0} \cong(2.722 \pm 0.027) \mathrm{K}$.

2) According to the advanced observational data analysis by Riess et al.(2016) current best value of $H_{0} \cong(73.24 \pm 1.74) \mathrm{km} / \mathrm{sec} / \mathrm{Mpc}$.

3) With reference to $T_{0} \cong 2.722 \mathrm{~K}$ and our proposed set of concepts, in this paper, we choose, $H_{0} \cong 70 \mathrm{~km} / \mathrm{sec} / \mathrm{Mpc} \cong 2.26853 \times 10^{-18} \mathrm{sec}^{-1}$. This value seems to lie in between $(67.31$ and 73.24$) \mathrm{km} / \mathrm{sec} / \mathrm{Mpc}$.

\subsection{The Planck Scale in Entire Cosmic Evolution}

So far no mainstream cosmological model implemented Planck scale in current cosmic evolution. In this complicated situation, in a positive approach, we make an attempt to implement the 'Planck scale' in the entire cosmic evolution. With further study, our approach can be developed for a better understanding. Based on quantum gravity, we define the Planck scale Hubble parameter, $H_{p l} \cong \sqrt{\frac{c^{5}}{G \hbar}} \cong 1.854921 \times 10^{43} \mathrm{sec}^{-1}$. To proceed further, we define that,

$$
\left.\begin{array}{l}
\sqrt{\left(\frac{3 H_{t}^{2} c^{2}}{8 \pi G\left(a T_{t}^{4}\right)}\right)} \cong \gamma_{t} \cong\left[1+\ln \left(\frac{H_{p l}}{H_{t}}\right)\right] \\
\left(\frac{3 H_{t}^{2} c^{2}}{8 \pi G\left(a T_{t}^{4}\right)}\right)^{\frac{1}{4}} \cong \sqrt{\gamma_{t}} \cong \sqrt{1+\ln \left(\frac{H_{p l}}{H_{t}}\right)} \cong\left(\left(Z_{T}\right)_{t}+1\right)
\end{array}\right\}
$$

Based on this relation, if defined $H_{p l} \cong 1.854921 \times 10^{43} \mathrm{sec}^{-1}$, one can choose different values of $\gamma$ in between $\gamma_{p l} \cong 1$ and $\gamma_{0} \cong 141.2564$. For each assumed value of $H$, one can get a corresponding $\gamma$ and all other physical parameters can be estimated. For the Planck scale, $\sqrt{\left(\frac{3 H_{p l}^{2} c^{2}}{8 \pi G\left(a T_{p l}^{4}\right)}\right)} \cong \gamma_{p l} \cong 1$ and for the current case, $\sqrt{\left(\frac{3 H_{0}^{2} c^{2}}{8 \pi G\left(a T_{0}^{4}\right)}\right)} \cong \gamma_{0} \cong\left[1+\ln \left(\frac{H_{p l}}{H_{0}}\right)\right] \cong 141.2564$. In a simplified form, cosmic temperature can be expressed as,

$$
T_{t} \cong \frac{1}{\sqrt{\gamma_{t}}}\left(\frac{3 H_{t}^{2} c^{2}}{8 \pi G a}\right)^{\frac{1}{4}} \cong \frac{\left(T_{c}\right)_{t}}{\sqrt{\gamma_{t}}} \cong \frac{1}{\sqrt{\gamma_{t}}}\left\{\frac{0.652632 \hbar \sqrt{H_{p l} H_{t}}}{k_{B}}\right\}
$$

\section{TO ESTIMATE THE COSMIC MASS, RADIUS AND EXPANSION VELOCITY}

$$
\text { Let, } \begin{aligned}
\left(M_{O M}+M_{D M}\right)_{t} \cong M_{t} \cong\left[\left(\Omega_{O M}\right)_{t}+\left(\Omega_{D M}\right)_{t}\right]\left(\frac{3 H_{t}^{2}}{8 \pi G}\right)\left(\frac{4 \pi}{3} R_{t}^{3}\right) \\
G\left(M_{O M}+M_{D M}\right)_{t} \cong G M_{t} \cong R_{t} c^{2}
\end{aligned}
$$




$$
R_{t} \cong \frac{G\left(M_{O M}+M_{D M}\right)_{t}}{c^{2}} \cong \frac{G M_{t}}{c^{2}}
$$

Based on these relations, it is possible to show that,

$$
\begin{gathered}
R_{t} \cong \frac{G\left(M_{O M}+M_{D M}\right)_{t}}{c^{2}} \cong \sqrt{\frac{2}{\left(\Omega_{O M}+\Omega_{D M}\right)_{t}}}\left(\frac{c}{H_{t}}\right) \\
\left(V_{\text {exp }}\right)_{t} \cong R_{t} H_{t} \cong \sqrt{\frac{2}{\left(\Omega_{O M}+\Omega_{D M}\right)_{t}}}(c) \\
\left(M_{O M}+M_{D M}\right)_{t} \cong \sqrt{\frac{2}{\left(\Omega_{O M}+\Omega_{D M}\right)_{t}}\left(\frac{c^{3}}{G H_{t}}\right) \cong\left(\frac{c^{2}\left(V_{\text {exp }}\right)_{t}}{G H_{t}}\right)}
\end{gathered}
$$

Based on relations (6 to 8$)$ and in terms of $\left(\Omega_{O M}\right)_{t}$ and $\left(\Omega_{D M}\right)_{t}$,

$$
\begin{aligned}
& \left(M_{O M}\right)_{t} \cong\left[\frac{\left(\Omega_{O M}\right)_{t}}{\left(\Omega_{O M}+\Omega_{D M}\right)_{t}}\right]\left(\frac{c^{2}\left(V_{\exp }\right)_{t}}{G H_{t}}\right) \\
& \left(M_{D M}\right)_{t} \cong\left[\frac{\left(\Omega_{D M}\right)_{t}}{\left(\Omega_{O M}+\Omega_{D M}\right)_{t}}\right]\left(\frac{c^{2}\left(V_{\exp }\right)_{t}}{G H_{t}}\right)
\end{aligned}
$$

\section{UNDERSTANDING THE TREND OF ORDINARY AND DARK MATTER DENSITY RATIOS}

With the help of defined $\gamma_{t} \cong\left[1+\ln \left(\frac{H_{p l}}{H_{t}}\right)\right]$ and $\sqrt{\gamma_{t}} \cong \sqrt{1+\ln \left(\frac{H_{p l}}{H_{t}}\right)}$, on ad-hoc basis and with reference to current observed values of $\left(\Omega_{O M}\right)_{0}$ and $\left(\Omega_{D M}\right)_{0}$, we are making an attempt to estimate the past values of $\left(\Omega_{O M}\right)_{t}$ and $\left(\Omega_{D M}\right)_{t}$. In this context, the basic question to be answered is: Is there any scope for the existence for dark matter at Planck scale? It needs further study.

\subsection{About ordinary matter density ratio}

If it is assumed that,

$$
\left\{\left[\left(M_{O M}\right)_{t} c^{2}\right] /\left[\frac{4 \pi}{3} R_{t}^{3}\right]\right\} \propto \sqrt{\left(\frac{3 H_{t}^{2} c^{2}}{8 \pi G}\right)\left(a T_{t}^{4}\right)}
$$

As per relation (1),

$$
\left\{\left[\left(M_{O M}\right)_{t} c^{2}\right] /\left[\frac{4 \pi}{3} R_{t}^{3}\right]\right\} \propto \frac{1}{\gamma_{t}}\left(\frac{3 H_{t}^{2} c^{2}}{8 \pi G}\right)
$$

By considering an ad-hoc proportionality coefficient of the form, $\beta_{0} \cong\left(\frac{1+\sqrt{\gamma_{0}}}{2}\right) \cong\left(1+\frac{\left(Z_{T}\right)_{0}}{2}\right)$,

$$
\begin{gathered}
\left\{\left[\left(M_{O M}\right)_{0} c^{2}\right] /\left[\frac{4 \pi}{3} R_{0}^{3}\right]\right\} \cong \frac{\beta_{0}}{\gamma_{0}}\left(\frac{3 H_{0}^{2} c^{2}}{8 \pi G}\right) \\
\text { and }\left(\Omega_{O M}\right)_{0} \cong\left(\frac{1}{\gamma_{0}}\right)\left(\frac{1+\sqrt{\gamma_{0}}}{2}\right) \cong \frac{\beta_{0}}{\gamma_{0}} \cong 0.04561 \\
\text { where } \sqrt{\gamma_{0}} \cong\left[1+\left(Z_{T}\right)_{0}\right] \cong\left(1+Z_{2.722 K}\right) \cong 11.885 \text { and } Z_{2.722 K} \cong 10.885 .
\end{gathered}
$$




\subsection{About dark matter density ratio}

Like the case of ordinary matter density,

$$
\left\{\left[\left(M_{D M}\right)_{t} c^{2}\right] /\left[\frac{4 \pi}{3} R_{t}^{3}\right]\right\} \propto \sqrt{\left(\frac{3 H_{t}^{2} c^{2}}{8 \pi G}\right)\left(a T_{t}^{4}\right)}
$$

As per relation (1),

$$
\left\{\left[\left(M_{D M}\right)_{t} c^{2}\right] /\left[\frac{4 \pi}{3} R_{t}^{3}\right]\right\} \propto \frac{1}{\gamma_{t}}\left(\frac{3 H_{t}^{2} c^{2}}{8 \pi G}\right)
$$

By considering an ad-hoc proportionality coefficient of the form, $\beta_{0}^{2} \cong\left(\frac{1+\sqrt{\gamma_{0}}}{2}\right)^{2} \cong\left(1+\frac{\left(Z_{T}\right)_{0}}{2}\right)^{2}$,

$$
\begin{aligned}
& \left\{\left[\left(M_{D M}\right)_{0} c^{2}\right] /\left[\frac{4 \pi}{3} R_{0}^{3}\right]\right\} \cong \frac{\beta_{0}^{2}}{\gamma_{0}}\left(\frac{3 H_{0}^{2} c^{2}}{8 \pi G}\right) \\
& \text { and }\left(\Omega_{D M}\right)_{0} \cong\left(\frac{1}{\gamma_{0}}\right)\left(\frac{1+\sqrt{\gamma_{0}}}{2}\right)^{2} \cong \frac{\beta_{0}^{2}}{\gamma_{0}} \cong 0.29384
\end{aligned}
$$

\subsection{General expressions for understanding the trend of ordinary and dark matter density ratios}

By extrapolating the coefficient to past and future as,

$$
\left.\begin{array}{c}
\beta_{t} \cong\left(\frac{1+\sqrt{\gamma_{t}}}{2}\right) \text { and } \beta_{t}^{2} \cong\left(\frac{1+\sqrt{\gamma_{t}}}{2}\right)^{2} \\
\left.\mathrm{~A})\left(\Omega_{O M}\right)_{t} \cong\left(\frac{1}{\gamma_{t}}\right)\left(\frac{1+\sqrt{\gamma_{t}}}{2}\right) \cong \frac{\beta_{t}}{\gamma_{t}} ; \mathrm{B}\right)\left(\Omega_{D M}\right)_{t} \cong\left(\frac{1}{\gamma_{t}}\right)\left(\frac{1+\sqrt{\gamma_{t}}}{2}\right)^{2} \cong \frac{\beta_{t}^{2}}{\gamma_{t}} \\
\text { C) } \left.\frac{\left(\Omega_{D M}\right)_{t}}{\left(\Omega_{O M}\right)_{t}} \cong\left(\frac{1+\sqrt{\gamma_{t}}}{2}\right) \cong \beta_{t} ; \mathrm{D}\right)\left(\Omega_{O M}+\Omega_{D M}\right)_{t} \cong \frac{\beta_{t}+\beta_{t}^{2}}{\gamma_{t}}
\end{array}\right\}
$$

See the following picture-1. With reference to critical density, green curve represents the trend of ordinary matter density ratio and black curve represents the trend of dark matter density ratio.

Figure 1: Decreasing trend of ordinary and dark matter density ratios

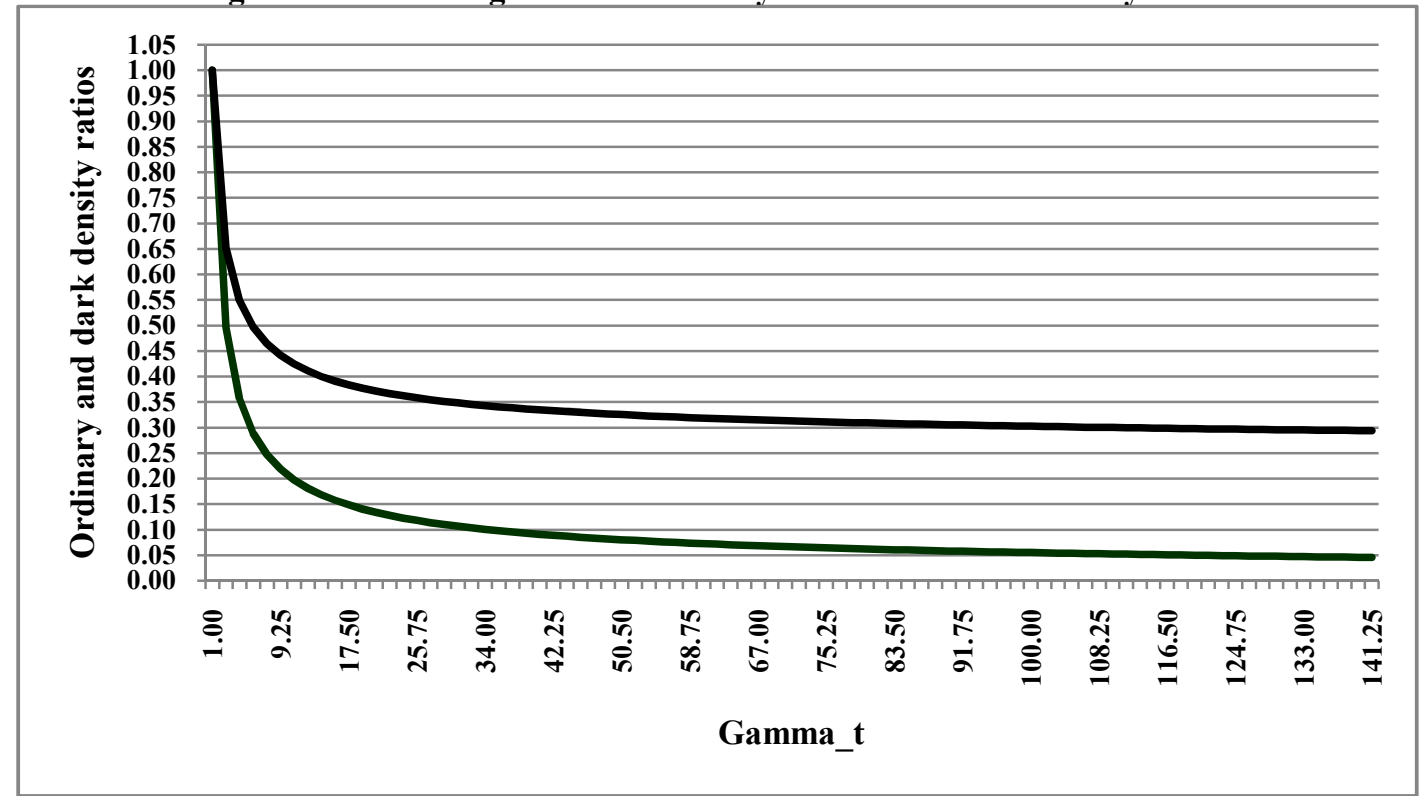


Qualitatively, we noticed that, $\frac{\left(Z_{T}\right)_{t}}{2} \approx \ln \left(\gamma_{t}\right)$ and $\beta_{t} \approx 1+\ln \left(\gamma_{t}\right)$. With reference to the 'status of existence' of ordinary matter and dark matter and with further research, various relations for $\beta_{t}$ can be developed using by which, in near future, trend of ordinary and dark matter density ratios can be understood right from the beginning of Planck scale. See figure -2 for the drcreasing trend of total matter density ratio.

Figure 2: Decreasing trend of total matter density ratio

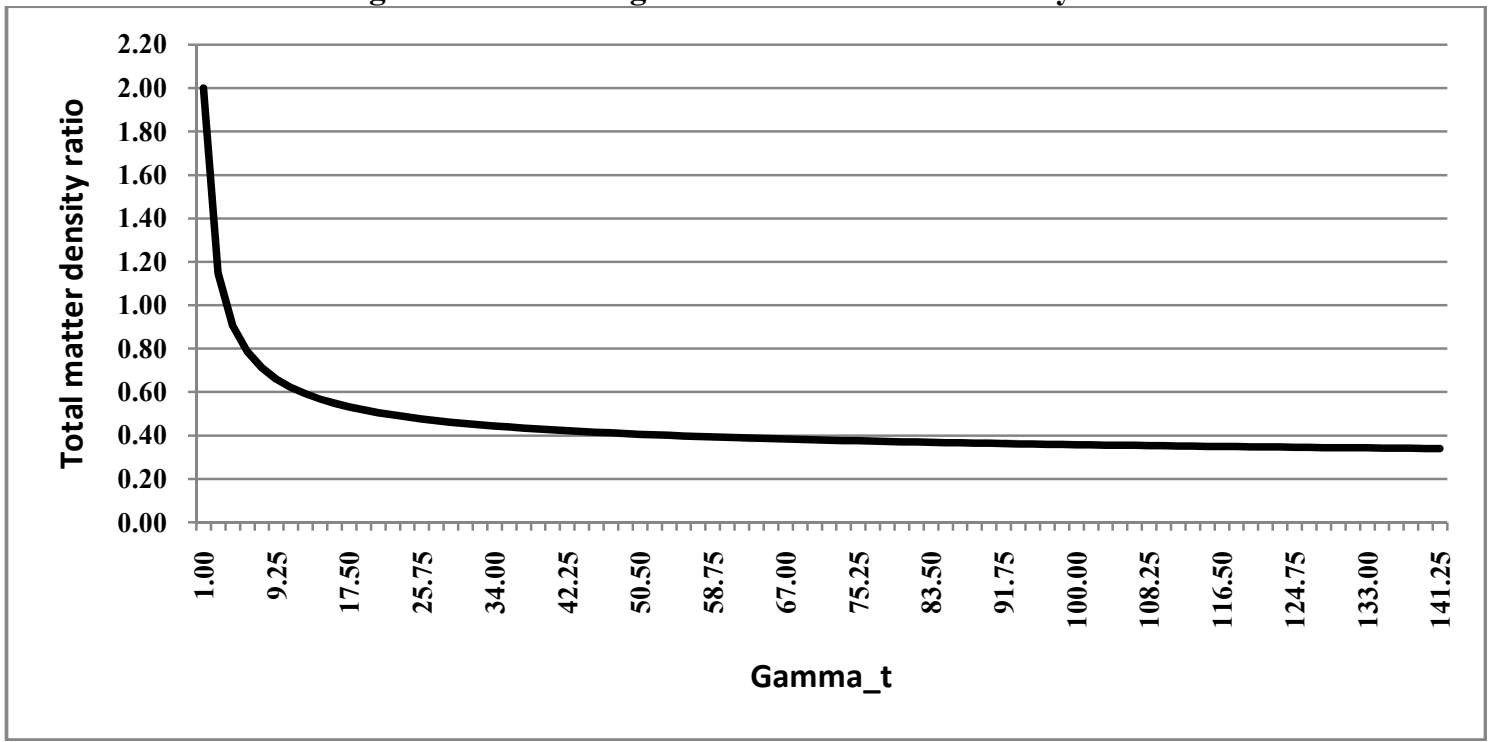

\section{CURRENT AND PLANCK SCALE COSMIC PHYSICAL PARAMETERS}

See table-1 for various cosmic physical parameters associated with current and Planck scales.

Table-1: Current and Planck scale cosmic physical parameters

\begin{tabular}{|l|l|}
\hline \multicolumn{1}{|c|}{ Current scale } & \multicolumn{1}{c|}{ Planck scale } \\
\hline$H_{0} \cong 70 \mathrm{~km} / \mathrm{sec} / \mathrm{Mpc} \cong 2.26853 \times 10^{-18} \mathrm{sec}^{-1}$ & $H_{p l} \cong \sqrt{\frac{c^{5}}{G \hbar}} \cong 1.855 \times 10^{43} \mathrm{sec}^{-1}$ \\
\hline$\gamma_{0} \cong\left[1+\ln \left(\frac{H_{p l}}{H_{0}}\right)\right] \cong 141.2564$ & $\gamma_{p l} \cong\left[1+\ln \left(\frac{H_{p l}}{H_{p l}}\right)\right] \cong 1$ \\
\hline$\left(\Omega_{O M}\right)_{0} \cong 0.04561$ & $\left(\Omega_{O M}\right)_{p l} \cong 1$ \\
\hline$\left(\Omega_{D M}\right)_{0} \cong 0.29384$ & $\left(\Omega_{D M}\right)_{p l} \cong 1$ \\
\hline$T_{0} \cong\left(\frac{1}{\sqrt{\gamma_{0}}}\right)\left(\frac{3 H_{0}^{2} c^{2}}{8 \pi G a}\right)^{\frac{1}{4}} \cong 2.721 \mathrm{~K}$ & $T_{p l} \cong\left(\frac{1}{\sqrt{\gamma_{p l}}}\right)\left(\frac{3 H_{p l}^{2} c^{2}}{8 \pi G a}\right)^{\frac{1}{4}} \cong 9.247 \times 10^{31} \mathrm{~K}$ \\
\hline$R_{0} \cong \sqrt{\left[\left(\Omega_{O M}\right)_{0}+\left(\Omega_{D M}\right)_{0}\right]} \frac{c}{H_{0}}$ & $R_{p l} \cong \sqrt{\frac{\left[\left(\Omega_{O M}\right)_{p l}+\left(\Omega_{D M}\right)_{p l}\right.}{2}}\left(\frac{c}{H_{p l}}\right)$ \\
$\cong 3.207 \times 10^{26} \mathrm{~m} \cong 10.40 \mathrm{Gpc}$ & $\cong 1.616 \times 10^{-35} \mathrm{~m}$ \\
\hline$\left(V_{\text {exp }}\right)_{0} \cong R_{0} H_{0} \cong 2.42654 c$ & $\left(V_{\text {exp }}\right)_{p l} \cong R_{p l} H_{p l} \cong c$ \\
\hline$\left(M_{O M}\right)_{0} \cong 5.836 \times 10^{52} \mathrm{~kg}$ & $\left(M_{O M}\right)_{p l} \cong 1.0882 \times 10^{-8} \mathrm{~kg}$ \\
\hline
\end{tabular}




\begin{tabular}{|l|l|}
\hline$\left(M_{D M}\right)_{0} \cong 3.752 \times 10^{53} \mathrm{~kg}$ & $\left(M_{D M}\right)_{p l} \cong 1.0882 \times 10^{-8} \mathrm{~kg}$ \\
\hline$\left[\left(M_{O M}\right)_{0}+\left(M_{D M}\right)_{0}\right] \cong 4.3352 \times 10^{53} \mathrm{~kg}$ & {$\left[\left(M_{O M}\right)_{p l}+\left(M_{D M}\right)_{p l}\right] \cong 2.176 \times 10^{-8} \mathrm{~kg}$} \\
\hline$t_{0} \cong \frac{2 R_{0}}{\left(\left(V_{\text {exp }}\right)_{0}+\left(V_{\text {exp }}\right)_{p l}\right)} \cong 19.78$ BillionYears & $t_{p l} \cong 0$ \\
\hline
\end{tabular}

\section{COSMIC SCALE FACTOR AND REDSHIFT}

With reference to the proposed relations (1) and (2) and with reference to the current definitions of cosmic redshift and scale factor, it is possible to show that,

$$
\begin{aligned}
& \left(\frac{1}{a} \cong(z+1) \cong \frac{T_{t}}{T_{0}}\right) \cong \sqrt{\frac{\gamma_{0} H_{t}}{\gamma_{t} H_{0}}} \cong \sqrt{\frac{\gamma_{0}}{\gamma_{t}}} \sqrt{\frac{H_{t}}{H_{0}}} \\
& \cong \sqrt{\frac{\gamma_{0}}{\gamma_{t}}}\left\{\exp \left(\frac{\gamma_{0}-\gamma_{t}}{2}\right)\right\} \cong \sqrt{\left(\frac{\gamma_{0}}{\gamma_{t}}\right) \exp \left(\gamma_{0}-\gamma_{t}\right)}
\end{aligned}
$$

Redshift can be expressed in the following form.

$$
z \cong \sqrt{\left(\frac{\gamma_{0}}{\gamma_{t}}\right) \exp \left(\gamma_{0}-\gamma_{t}\right)}-1
$$

We are working on interpreting this relation and it needs further study.

\section{TO ESTIMATE THE CURRENT COSMIC AGE}

From the beginning of cosmic evolution, based on the proposed cosmic expansion velocities, cosmic age can be approximated with the following relation.

$$
t \cong \frac{\left(R_{t}-R_{p l}\right)}{\left[\left(\left(V_{\exp }\right)_{t}+\left(V_{\text {exp }}\right)_{p l}\right) / 2\right]}
$$

where $\left[\left(\left(V_{\exp }\right)_{t}+\left(V_{\exp }\right)_{p l}\right) / 2\right]$ can be considered as average expansion velocity.

For the current case,

$$
t_{0} \cong \frac{\left(R_{0}-R_{p l}\right)}{\left[\left(\left(V_{\exp }\right)_{0}+\left(V_{\exp }\right)_{p l}\right) / 2\right]} \cong \frac{2 R_{0}}{\left(\left(V_{\exp }\right)_{0}+\left(V_{\text {exp }}\right)_{p l}\right)} \cong 19.78 \text { BillionYears }
$$

For a temperature of $3000 \mathrm{~K}$, it is possible to show that,

$$
\left.\begin{array}{l}
H_{3000 K} \cong 2.49 \times 10^{-12} \mathrm{sec}^{-1} \\
\gamma_{3000 K} \cong 1+\ln \left(\frac{H_{p l}}{H_{3000 K}}\right) \cong 127.34774 \\
Z_{3000 K} \cong \sqrt{\gamma_{3000 K}}-1 \cong 10.285 \\
z_{3000 K} \cong \sqrt{\frac{\gamma_{0}}{\gamma_{3000 K}} \exp \left(\gamma_{0}-\gamma_{3000 K}\right)}-1 \cong 1102.407
\end{array}\right\}
$$

Cosmic age corresponding to a temperature of $\mathrm{T}=3000 \mathrm{~K}$ can be estimated to be, 


$$
t_{3000 K} \cong \frac{\left(R_{3000 K}-R_{p l}\right)}{\left[\left(\left(V_{\text {exp }}\right)_{3000 K}+\left(V_{\text {exp }}\right)_{p l}\right) / 2\right]} \cong 17987.07 \text { Years }
$$

This estimation is 21.13 times less than the current estimations and needs further study.

\section{VELOCITY-DISTANCE RELATION}

From and about the hypothetical baby Planck ball or baby universe, current galactic receding speeds can be approximated with,

$$
\left(v_{g}\right)_{0} \cong\left(\frac{\left(d_{g}\right)_{0}}{R_{0}}\right)\left(V_{\text {exp }}\right)_{0} \cong\left(\frac{\left(V_{\text {exp }}\right)_{0}}{R_{0}}\right)\left(d_{g}\right)_{0} \cong H_{0}\left(d_{g}\right)_{0}
$$

When $\left(d_{g}\right)_{0} \rightarrow R_{0},\left(v_{g}\right)_{0} \cong H_{0} R_{0}$. This can be compared with currently believed Hubble's law for the current expanding universe.

\section{DISCUSSION}

\subsection{Cosmological Constant Problem}

With reference to proposed concepts, ratio of the Planck scale critical density to the current critical density is,

$$
\left(\frac{3 H_{p l}^{2} c^{2}}{8 \pi G}\right) \div\left(\frac{3 H_{0}^{2} c^{2}}{8 \pi G}\right) \cong\left(\frac{H_{p l}}{H_{0}}\right)^{2} \cong 6.685 \times 10^{121}
$$

We wish to appeal that, this idea can be considered as a characteristic tool for constructing a model of 'quantum gravity' with cosmic evolution.

\subsection{Horizon Problem}

The 'horizon problem' is a problem with the standard cosmological model of the Big Bang. It points out that different regions of the universe have not 'contacted' each other because of the large distances between them, but nevertheless they have the same temperature and other physical properties. If one is willing to consider the concept of 'matter causes the space-time to curve', 'horizon problem' can be understood. According to hot big bang model, during its evolution, as the universe is expanding, thermal radiation temperature decreases and matter content increases. As matter content increases, based on Mach's principle, at any stage of evolution, it is possible to have an increasing radius of curvature, $R_{t} \cong \frac{G}{c^{2}}\left[\left(M_{O M}\right)_{t}+\left(M_{D M}\right)_{t}\right]$. For the current case, $R_{0} \cong \frac{G}{c^{2}}\left[\left(M_{O M}\right)_{0}+\left(M_{D M}\right)_{0}\right] \cong 10.40 \mathrm{Gpc}$ and there is no scope for 'causal disconnection' of distant visible matter.

\subsection{Cosmic Inflation}

Basically, 'inflation' (Guth 1981, Linde 1982) is a period of super cooled cosmic expansion, where temperature seems to be dropped by a factor of 100,000 and is mostly model dependent. Mainstream cosmologists believe that just after the hot big bang, 'inflation' seems to be started at $10^{-37}$ seconds and ended by $10^{-32}$ seconds. During this time span, cosmic temperature seems to be dropped from $10^{27} \mathrm{~K}$ to $10^{22} \mathrm{~K}$. Since that time, expansion initially decelerated and then, after about 6 billion years, began very slowly to accelerate. Most puzzling point to be noted is that, the detailed mechanism responsible for inflation is still unknown. Many cosmologists proposed different starting mechanisms for initiating and fine tuning the believed 'inflation'. Since its introduction by Alan Guth in 1980, the inflationary paradigm has become widely accepted. Currently, a great debate is going on its existence (Steinhardt 2011, Ijjas et al 2014). In this proposed 
model, when $H_{t} \approx \frac{1}{t} \approx 10^{37} \mathrm{sec}^{-1}$, estimated $\left(\gamma_{t} \cong 15.43, T_{t} \cong 1.73 \times 10^{28} \mathrm{~K}\right)$. Similarly, when $H_{t} \approx \frac{1}{t} \approx 10^{32} \mathrm{sec}^{-1}$, estimated $\left(\gamma_{t} \cong 26.946, T_{t} \cong 4.14 \times 10^{25} \mathrm{~K}\right)$. Thus, for the currently believed inflationary time span of $\left(10^{-37}\right.$ to $\left.10^{-32}\right) \mathrm{sec}$, estimated ratio of initial and final temperatures seems to be around 400 and needs further study with respect to the believed ratio of 100000 . We are working in this direction.

\subsection{Cosmic Acceleration and Expansion Velocity}

Before 1990s, most of the cosmologists thought that the cosmic expansion would be decelerating due to the gravitational attraction of the matter in the universe. In between 1995 and 1998, as space technology was advancing, based on the available supernovae redshift data, cosmologists noticed that, rather than slowing down, universe was expanding with increasing rate (Riess et al. 1998, Perlmutter et al. 1999). Even though it was a shocking news for most of the science community, confirmatory evidence has been found in baryon acoustic oscillations and other cosmological observations (Weinberg DH. et al, 2013). Considering the updated supernovae redshift data, in 2016, cosmologists noticed that, universe is coasting at constant speed rather than acceleration (Neilsen et al, 2016). In this context, now a days, a great debate has been initiated among various groups of cosmologists on 'cosmic acceleration' (Zhengxiang Li et al, 2011 ; D. Rubin and B. Hayden, 2016; Tutusaus et al, 2018; Lawrence H. Dam et al, 2017; Christos G Tsagas, 2011)

Now a days, main stream cosmologists are seriously working on 'eternal light speed expansion' (John 2016, Jun-Jie Wei, et al 2015, Melia 2012, Melia et al 2016). In this context, in our earlier published papers, based on ordinary matter density and Hubble's law, we come across different magnitudes of cosmic expansion velocities ranging from $2 c$ to $12 c$. We would like to suggest that, by considering a decreasing trend of ordinary matter and dark matter density, starting from the Planck scale, it is possible to get an expression for cosmic expansion velocity comparable to speed of light. It can be expressed as follows.

$$
\frac{\left(V_{\exp }\right)_{t}}{c} \cong \sqrt{\frac{2}{\left[\left(\Omega_{O M}\right)_{t}+\left(\Omega_{D M}\right)_{t}\right]}}
$$

Based on this expression, for the Planck scale, $\left(V_{\exp }\right)_{p l} \cong c$ and for the current scale, $\left(V_{\text {exp }}\right)_{0} \cong 2.427 c$. Interesting point to be noted is that, after 20 billion years of cosmic expansion, increment in expansion velocity seems to be only $\left[\left(V_{\exp }\right)_{0}-\left(V_{\exp }\right)_{p l}\right] \cong 1.427 c$. See the following picture-3.

Figure 3: Increasing trend of cosmic expansion velocity

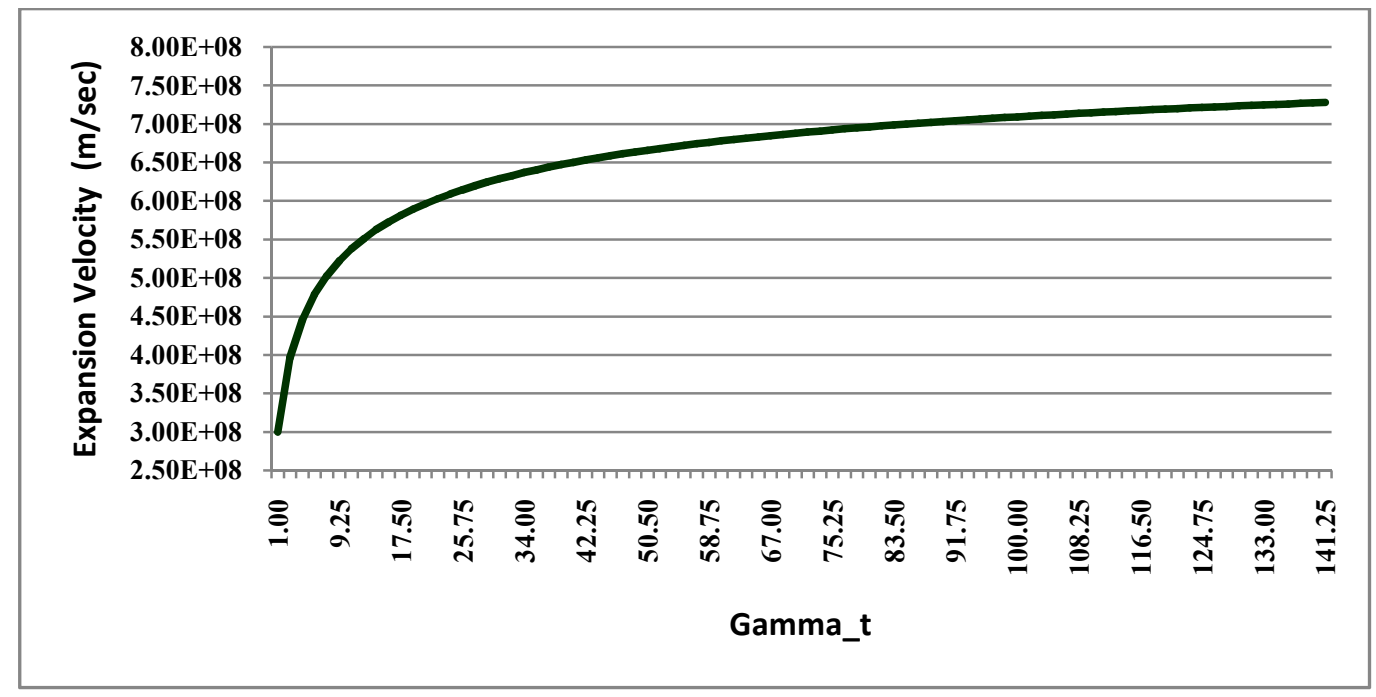

From figure-3, it is very clear that, right from the beginning of cosmic evolution, cosmic expansion velocity seems have an increasing trend. To some extent, this can be compared with currently believed cosmic acceleration concept. 
Interesting point to be noted is that, expansion velocity seems to depend on $\sqrt{\frac{2}{\left[\left(\Omega_{O M}\right)_{t}+\left(\Omega_{D M}\right)_{t}\right]}}$. In near future, if decrease in $\left[\left(\Omega_{O M}\right)_{t}+\left(\Omega_{D M}\right)_{t}\right]$ is found to be significant, one can expect 'acceleration' and if decrease in $\left[\left(\Omega_{O M}\right)_{t}+\left(\Omega_{D M}\right)_{t}\right]$ is found to be insignificant, one can expect cosmic 'constant rate of expansion'. It is for further study.

\section{CONCLUSIONS}

As current observational status of 'quantum cosmology' is very poor (Bojowald 2015), we would like to suggest that:

1) Universe can be considered as a quantum gravitational object.

2) Planck mass can be considered as a characteristic representation of the mass of baby universe.

3) Mach's relation can be given some consideration in developing a workable model of cosmology.

4) Proposed temperature redshift $\left(Z_{T}+1\right)$ assumed to be associated with $\sqrt{1+\ln \left(\frac{H_{p l}}{H_{t}}\right)}$ can be recommended for further research. For the current case, $\left(Z_{2.722 K}+1\right) \cong 11.885$ and $Z_{2.722 K} \cong 10.885$.

5) Even though it is very interesting, it is also true that the proposed relation $\gamma_{t} \cong\left[1+\ln \left(\frac{H_{p l}}{H_{t}}\right)\right] \cong \sqrt{\frac{3 H_{t}^{2} c^{2}}{8 \pi G\left(a T_{t}^{4}\right)}}$ is new and peculiar. We are confident that, its role, scope and validity can be verified with further study.

6) Estimated current cosmic Planck ball radius is about $10.40 \mathrm{Gpc}$ and can be compared with current observations of $14 \mathrm{Gpc}$. Current cosmic sphere seems to constitute around 14 Hubble spheres and needs further study with respect to the Bayesian model average estimate of $>251$ Hubble spheres proposed by Vardanyan et al (2011).

7) With reference to ordinary matter density ratio, dark matter density ratio and the proposed relation $\left(\frac{3 H_{t}^{2} c^{2}}{8 \pi G\left(a T_{t}^{4}\right)}\right)^{\frac{1}{4}} \cong \sqrt{\gamma_{t}} \cong \sqrt{1+\ln \left(\frac{H_{p l}}{H_{t}}\right)} \cong\left(\left(Z_{T}\right)_{t}+1\right)$, we are working on understanding the scope, physical significance and validity of the proposed ad-hoc coefficients, $\beta_{t} \cong\left(\frac{1+\sqrt{\gamma_{t}}}{2}\right) \cong\left(1+\frac{\left(Z_{T}\right)_{t}}{2}\right)$ and $\beta_{t}^{2} \cong\left(\frac{1+\sqrt{\gamma_{t}}}{2}\right)^{2} \cong\left(1+\frac{\left(Z_{T}\right)_{t}}{2}\right)^{2}$.

8) Independent of galactic redshift data, we are working on finding alternative tools for understanding the cosmic expansion rate. In future, with advanced science, engineering and technology, by considering $\frac{d\left(T_{0}\right)}{d t}$ or $\frac{d\left(H_{0}\right)}{d t}$ or $\frac{d\left(\Omega_{O M}+\Omega_{D M}\right)_{0}}{d t}$, absolute cosmic rate of expansion can be estimated.

\section{ACKNOWLEDGEMENTS}

It is great pleasure to express our deep gratitude to Dr.E.Terry Tatum for his valuable technical discussions and kind encouragement. Author Seshavatharam is indebted to professors brahmashri M. Nagaphani Sarma, Chairman, shri K.V. Krishna Murthy, founder Chairman, Institute of Scientific Research in Vedas (I-SERVE), Hyderabad, India and Shri K.V.R.S. Murthy, former scientist IICT (CSIR), Govt. of India, Director, Research and Development, I-SERVE, for their valuable guidance and great support in developing this subject.

\section{REFERENCES}

Abhas Mitra, 2014, MNRAS 442, 382-387

Abhas Mitra, 2011, Journal of Modern Physics, 2, 1436-1442

Annila A, 2012, Mon. Not. R. Astron. Soc. 423, 1973-1977

Arbab. A.I, 2001, Spacetime \& Substance, Vol. 2, No. 2 (7), pp. 55-62

Arbab. A.I, 2004, Gen.Rel.Grav.36:2465-2479. 
Balakrishna S. Haridasu et al. 2017, A\&A 600, L1

Berman MS, 2008, Astrophys.SpaceSci.318:269-272, 2008

Berman MS, 2009, Int J Theor Phys 48: 3257-3261

Bojowald M, 2015, Rep. Prog. Phys. 78, 023901.

Christos G Tsagas, 2011, J. Phys.: Conf. Ser. 283012040 (2011)

D. Rubin and B. Hayden, 2016, The Astrophysical Journal Letters, 833:L30 (5pp).

Deng Wang and Xin-He Meng, 2017, Phys. Rev. D 96, 103516

Einstein A, 1918, Ann. Phys. 360, 241.

Gamow G, 1948, Nature, 162, 680-682.

Gamow G, 1948, Phys. Rev. 74, 505-506

G'abor R'acz et al. 2017, Mon Not R Astron Soc Lett slx026.

Gong-Bo Zhao et al, 2017, Nature Astronomy, 2017; 1 (9): 627

Guth. A.H, 1981, Phys. Rev.;D23:347.

Ijjas A, Steinhardt P. J and Abraham Loeb. 2014, Physics Letters B 736, 142-146.

Joel Smoller et al, 2017, Proceedings of the Royal Society A: Mathematical, Physical and Engineering Science John M.V. 2016, Mon.Not.Roy.Astron.Soc. 000: 1-12.

Jun-Jie Wei, et al, 2015, The Astronomical Journal, 149:102 (11pp).

Jungjai Lee and Hyun Seok Yang., 2017. Preprint (arXiv:1709.04914)

Lasha Berezhiani et al,2017, Phys. Rev. D 95, 123530.

Lawrence H. Dam et al., 2017, Mon.Not.Roy.Astron.Soc, 472 (2017) 835-851

Linde A. 1982, Physics Letters B. 108 (6): 389-393.

Miyazaki A, 2001, arXiv:gr-qc/0101112v1

Melia F. 2012, Astron. J. 144.

Melia F, et al.2016,Mon.Not.Roy.Astron.Soc. 456(4): 3422-3431.

Neilsen J.T. et al., 2016, Scientific Reports 6: 35596.

Padmanabhan T., 2005, Curr.Sci.88:1057.

Patrick Grahn et al, 2018, AIP Advances 8, 035028

Perlmutter S et al. 1999, Astrophys.J.517:565-586

Planck Collaboration: Planck 2015 Results. XIII. Cosmological Parameters.

Rennan Barkana, 2018. Nature 555 71-74

Riess AG et al. 1998, Astron.J.116:1009-1038.

Riess AG et al. 2016, Astrophys.J. 826 no.1.

Seshavatharam U. V. S \& Lakshminarayana S, 2017, Physical Science International Journal,14(2):1-17.

Seshavatharam U. V. S \& Lakshminarayana S, 2017, Physical Science International Journal 15(4): 1-13.

Seshavatharam U. V. S \& Lakshminarayana S, 2018, International Journal of Astronomy, Astrophysics and Space Science, 5(1), 1-13

Seshavatharam U. V. S \& Lakshminarayana. S, 2018, Prespacetime journal, Vol 9, Issue 4, Page 326-342.

Sivaram C., 2000, Current Science, Vol. 79, No. 4, 41-420, 25.

Steinhardt. P.J., 2011, Scientific American.;304(4):18-25.

Tatum, E.T et al. (2015) Journal of Applied Physical Science International, 4, 18-26.

Tutusaus I et al, 2018. arXiv:1803.06197 [astro-ph.CO]

Vardanyan M. et al., 2011. MNRAS Lett 413, 1, L91-L95.

Weinberg DH et al, 2013, Physics Reports 530, 87-255.

Zhengxiang Li et al, 2011, Phys.Lett.B695:1-8. 